\title{
Size of a plasma cloud matters
}

\section{The polarisation electric field of a small-scale comet ionosphere}

\author{
H. Nilsson ${ }^{1,2}$, H. Gunell ${ }^{3,4}$, T. Karlsson ${ }^{5}$, N. Brenning ${ }^{5}$, P. Henri' ${ }^{6}$, C. Goetz ${ }^{7}$, A. I. Eriksson ${ }^{8}$, E. Behar ${ }^{1,2}$, \\ G. Stenberg Wieser ${ }^{1}$, and X. Vallières ${ }^{6}$ \\ 1 Swedish Institute of Space Physics, PO Box 812, 98128 Kiruna, Sweden \\ e-mail: hans.nilsson@irf.se \\ ${ }^{2}$ Luleå University of Technology, Department of Computer Science, Electrical and Space Engineering, Rymdcampus 1, 98128 \\ Kiruna, Sweden \\ 3 Belgian Institute for Space Aeronomy, Avenue Circulaire 3, 1180 Brussels, Belgium \\ 4 Department of Physics, Umeå University, 90187 Umeå, Sweden \\ 5 Department of Space and Plasma Physics, School of Electrical Engineering and Computer Science, KTH Royal Institute of Tech- \\ nology, Stockholm, Sweden \\ ${ }^{6}$ Laboratoire de Physique et Chimie de l'Environnement et de l'Espace (LPC2E), UMR 7328 CNRS - Université d'Orléans, France \\ 7 Technische Universität Braunschweig, Institute for Geophysics and Extraterrestrial Physics, Mendelssohnstraße 3, 38106 \\ Braunschweig, Germany \\ 8 Swedish Institute of Space Physics, Ångström Laboratory, Lägerhyddsvägen 1, Uppsala, Sweden
}

Received 10 April 2018 / Accepted 9 May 2018

\begin{abstract}
Context. The cometary ionosphere is immersed in fast flowing solar wind. A polarisation electric field may arise for comets much smaller than the gyroradius of pickup ions because ions and electrons respond differently to the solar wind electric field.

Aims. A situation similar to that found at a low activity comet has been modelled for barium releases in the Earth's ionosphere. We aim to use such a model and apply it to the case of comet 67P Churyumov-Gerasimenko, the target of the Rosetta mission. We aim to explain the significant tailward acceleration of cometary ions through the modelled electric field.

Methods. We obtained analytical solutions for the polarisation electric field of the comet ionosphere using a simplified geometry. This geometry is applicable to the comet in the inner part of the coma as the plasma density integrated along the magnetic field line remains rather constant. We studied the range of parameters for which a significant tailward electric field is obtained and compare this with the parameter range observed.

Results. Observations of the local plasma density and magnetic field strength show that the parameter range of the observations agree very well with a significant polarisation electric field shielding the inner part of the coma from the solar wind electric field. Conclusions. The same process gives rise to a tailward directed electric field with a strength of the order of $10 \%$ of the solar wind electric field.Using a simple cloud model we have shown that the polarisation electric field, which arises because of the small size of the comet ionosphere as compared to the pick up ion gyroradius, can explain the observed significant tailward acceleration of cometary ions and is consistent with the observed lack of influence of the solar wind electric field in the inner coma.
\end{abstract}

Key words. plasmas - acceleration of particles - comets: individual: 67P/Churyumov-Gerasimenko

\section{Introduction}

Early models employed to understand solar wind interaction with a comet atmosphere were based on experience from highly active and therefore large-scale comets, such as Halley (Neugebauer 1990), and employed methods mainly valid for large-scale phenomena. By large, we mean large compared to the gyroradius of cometary ions picked up in the solar wind flow. Examples include the model of field line draping at a comet by Alfvén (1957) and many others tested against the observations from comet Halley, e.g. Ogino et al. (1988) and Damas et al. (1994). The ESA mission Rosetta followed comet 67P over a large range of heliocentric distances, during which the comet ionosphere was small compared to a pick up ion gyroradius, but larger than the electron gyroradius. This presents a special challenge and the situation at such a smallscale comet has more similarities with the plasma dynamics of barium release experiments than with large-scale comets (Coates et al. 2015).

We employ a simple model developed to study barium release experiments (Brenning et al. 1991) and examine its usability to understand a small-scale comet environment such as that of comet 67P during most of the Rosetta mission. Our aim is to investigate the emergence and role of a polarisation electric field. The model is a complement to more detailed models (Koenders et al. 2016; Deca et al. 2017; Huang et al. 2018), where such polarisation electric fields may be difficult to reproduce, depending on how electron dynamics are implemented and owing to limited spatial resolution.

The atmosphere of a comet is peculiar in that it is not bound by the gravity of its parent body. Volatiles sublimating from the nucleus leave the surface with velocities above the escape velocity. The neutral atmosphere expands into surrounding space. The case of comet 67P during the Rosetta mission had a typical ra- 
dial velocity in the range 400-700 $\mathrm{m} \mathrm{s}^{-1}$ (Biver et al. 2015; Lee et al. 2015); see also discussion in Galand et al. (2016). The density of the expanding atmosphere falls off as $1 / r^{2}$, where $r$ is the distance to the comet, as confirmed experimentally with neutral gas pressure observations on the Rosetta spacecraft (Bieler et al. 2015; Hässig et al. 2015).

Solar extreme ultraviolet light, suprathermal electron and solar wind particle impact, and charge exchange collisions ionise part of the neutral atmosphere (Galand et al. 2016; Simon Wedlund et al. 2017), forming the ionosphere of the comet. As the atmosphere was continuously ionised, the ion density fell off as $1 / r$ for the position of Rosetta when Rosetta was located relatively close to the comet (Edberg et al. 2015; Nilsson et al. 2015a). Further out acceleration of the cometary particles changes the situation; at some point upstream of the nucleus the density can be expected to correspond to the local production and thus falls off as $1 / r^{2}$ (Behar et al.2018). We call the environment dominated by cometary ions, where the magnetic field is significantly enhanced as compared to the surrounding solar wind, the comet magnetosphere (Nilsson et al. 2015b; Cravens \& Gombosi 2004).

The plasma environment close to the comet is affected by collisions between ions and neutral particles. Rosetta was frequently in the vicinity of the point where the ion mean free path equals the distance to the nucleus (Mandt et al. 2016), termed the collisionopause or ion exobase. Thus ion collisions can be expected to have an effect on the plasma environment close to the nucleus. On the other hand a model estimate of the effect of charge exchange collisions on the acceleration of ions in an ambipolar radial electric field indicates that the collisions are not very efficient in coupling the ions to the neutrals (Vigren \& Eriksson 2017). Rosetta was typically well outside the electron collisionopause, as evidenced by the temperature of the electron population. Evidence for a warm electron population includes direct measurements and the mostly negative spacecraft potential (Eriksson et al. 2017; Odelstad et al. 2017).

The evolution of the ion environment with changing heliocentric distance has been summarised in several studies. Behar et al. (2017) showed the gradual evolution of the solar wind deflection expected due to the local acceleration of cometary ions and conservation of momentum. They further showed how the solar wind ion motion was consistent with a slowing down of the local electron fluid and a resulting gyration of the solar wind ions, which was later studied in more detail by Behar et al. (2018). Such a slowing down is consistent with the magnetic field in the local comet environment, which was enhanced as compared to the undisturbed solar wind (Goetz et al. 2017). Using data from an excursion on the dayside of the comet Goetz et al. (2017) showed that the magnetic field strength did not vary much with distance to the nucleus. Nilsson et al. (2017) showed the evolution of the cometary ion fluxes throughout the mission and noted that cometary ions were mainly moving anti-sunward. A study using a more limited data set, by Berčič et al. (2018), showed how ions accelerated to several $10 \mathrm{eV}$ energy and above were moving mainly antisunward, while the direction of the upstream solar wind electric field determined their motion in the plane perpendicular to the comet-sun line. Ions at lower energy were also moving with a significant anti-sunward component, but in the plane perpendicular to the comet-sun line they were moving radially away from the comet nucleus irrespective of the direction of the solar wind electric field. The latter implies that the inner part of the comet ionosphere is largely shielded from the main solar wind electric field because the ion motion is determined by an ambipolar electric field and another electric field giving rise to an anti-sunward motion.
Nilsson et al. (2015a, 2017) and Behar et al. (2016) noted that a polarisation electric field arising due to a different motion of non-magnetised ions and $\boldsymbol{E} \times \boldsymbol{B} / B^{2}$ drifting electrons could give rise to an anti-sunward electric field. The situation is similar to what has been described for the Martian tail by Dubinin et al. (1993), where it was noted that the situation of magnetised electrons and unmagnetised ions could lead to accelerated electrons dragging the ions with them by means of an electric field.

A similar situation has also been investigated for barium release experiments, where the initial cloud is small compared to the local ion gyroradius (Brenning et al. 1991; Haerendel 1982). We therefore aim to use the Brenning et al. (1991) cloud model to make a very simple estimate of the comet ionosphere-solar wind interaction. We discuss the shortcomings and the strengths of the model and compare results obtained with the model to observations by instruments in the Rosetta Plasma Consortium (RPC) instrument suite (Carr et al. 2007).

\section{Simple cloud model}

We recapitulate the simple cloud model presented by Brenning et al. (1991). Brenning et al. (1991) considered an injected plasma cloud (also known as a plasmoid) in motion through an ambient plasma. The plasma cloud was assumed to have the form of a cylinder with radius $R$ and length $L_{\|}$, where $L_{\|}$is along the magnetic field. The injected ions (the cometary ions for our purpose) have a constant uniform density $n_{\mathrm{C}}$, and all start at the time $t=0$ with the same velocity $V_{\mathrm{i}}$. At time $t$ $=0$ it is assumed that ions and electrons have the same distribution, such that charge neutrality is maintained everywhere in the studied volume. In this simple model, there is no further production after $t=0$. The gyroradius of the plasma cloud ions, for their given injection velocity, is assumed to be much larger than the plasma cloud radius, whereas the electron gyroradius is assumed to be small compared to the plasma cloud radius. The electrons thus move with the $\boldsymbol{E} \times \boldsymbol{B} / B^{2}$ drift. The plasma beta value is considered $\ll 1$, i.e. the plasma motion does not perturb the background magnetic field. The ambient plasma is assumed to have a density $n_{\mathrm{SW}}$ and an Alfvén velocity of $V_{\mathrm{A}}$. Collisions are not considered.

A further simplifying assumption is that all space charges, occurring because of the different dynamics of ions and electrons, are contained in a thin layer at the surface of the cylinder. The model does not consider instabilities at the boundary and it is assumed that field-aligned electric fields are negligible.

The processes we are interested in concern the separation of ions and electrons as they move across the magnetic field. Before proceeding, we should briefly consider the applicability of the model to the situation at a comet. Obviously, a comet does not have a uniform, cylindrically shaped ionosphere with no net production and plasma beta much less than one. However, as the size of the comet magnetosphere is much less than the cometary ion gyroradius in an unperturbed solar wind, at least for large heliocentric distances, the type of polarisation electric field that arises in the plasma cloud likely appears in the comet ionosphere as well. The comet ionosphere can be considered to be in motion in the solar wind reference frame with a uniform velocity. Plasma production is slow compared to the transport times involved in the build-up of a polarisation electric field. The role of the magnetic field in the model is primarily to determine the ion gyro radius and the electron $\boldsymbol{E} \times \boldsymbol{B} / B^{2}$ drift (see Sect. 3.2 for further discussion). Therefore this simple model can be used for a simple estimate of the polarisation electric field arising as the comet ionosphere moves through the ambient solar wind. We 
discuss the applicability in more detail once we have presented the model.

We define a coordinate system with $z$ along the plasma cylinder and thus also along the magnetic field, $x$ along the initial ion motion, and $y$ completing a right-handed system. We consider an initial displacement of ions with a density $n_{\mathrm{C}}$ a distance $\Delta x$ from the electrons. This gives no space charge in the interior of the cylinder, and the surface charge becomes

$\rho_{\mathrm{s}}=\rho_{0} \cos \phi$

where $\rho_{0}=e \Delta x n_{\mathrm{C}}, e$ is the unit charge, and $\phi$ is the azimuthal angle to the $x$ axis. Inside the cylinder this surface charge distribution gives rise to a uniform electric field,

$\boldsymbol{E}_{\text {in }}=\left(-\frac{1}{2} \frac{\rho_{0}}{\varepsilon_{0}}, 0,0\right)$,

while outside the cylinder we have the field from a line dipole (Herlofson 1951)

$\boldsymbol{E}_{\text {out }}=\frac{1}{2} \frac{\rho_{0}}{\varepsilon_{0}}\left[\frac{\left(x^{2}-y^{2}\right) R^{2}}{\left(x^{2}+y^{2}\right)^{2}}, \frac{2 x y R^{2}}{\left(x^{2}+y^{2}\right)^{2}}, 0\right]$.

Two currents inside the plasma cloud are associated with the electric field in this description: a current along the ion motion and a Hall current of the electrons due to their $E \times B$ drift. These currents accumulate charge at the surface, causing an electric field. The space charge accumulations at the cylinder surface caused by the different currents all cause a homogeneous electric field inside the cylinder and produce a line-dipole field outside. The total field obtained through superposition of the different charge accumulations is also uniform inside and produce a line dipole field outside of the cylinder. The change of the electric field is given by the change of the charge accumulation, i.e. given by the time derivative of Eq. (2). The contribution from the ions (index 1) and electrons (index 2) to the total charge accumulation along the cylinder length $L_{\|}$is given by

$\left(\frac{\mathrm{d} \boldsymbol{E}_{\mathbf{i n}}}{\mathrm{d} t} L_{\|}\right)_{1}=-\frac{1}{2} \frac{\boldsymbol{V}_{\boldsymbol{i}} n_{\mathrm{C}} e}{\varepsilon_{0}} L_{\|}$

$\left(\frac{\mathrm{d} \boldsymbol{E}_{\text {in }}}{\mathrm{d} t} L_{\|}\right)_{2}=\frac{1}{2} \frac{\boldsymbol{E}_{\text {in }} \times \boldsymbol{B}}{B^{2}} \frac{n_{\mathrm{C}} e}{\varepsilon_{0}} L_{\|}$,

where $\boldsymbol{B}$ is the local magnetic field strength.

The accumulated surface charges propagate along the field lines. The field-aligned currents caused by the charge accumulation may be determined using the Alfvén conductivity (Mallinckrodt \& Carlson 1978). The current density due to a perpendicular electric field in an Alfvén wave is given by

$i_{\|}=\frac{1}{\mu_{0} V_{\mathrm{A}}} E$

where $i_{\|}$is a field-aligned current in ambient space, which propagates in both directions along the magnetic field. The value $V_{\mathrm{A}}$ is the Alfvén velocity of ambient space. The electric field of the perturbation that propagates as an Alfvén wave is given by the divergence of the perpendicular electric field due to the charge accumulation at the cylinder surface, $\nabla \cdot \boldsymbol{E}_{\perp}$. For our case of a cylinder with a thin surface layer, this is $2 E_{\text {in }} \cos \phi$ as given by Eq. (1). Integrating Eq. (6) over the cylinder surface, we get the rate of change of the charge accumulation due to the fieldaligned currents
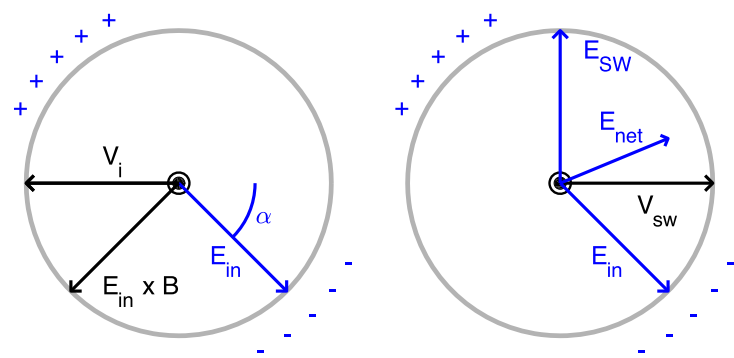

Fig. 1. Plasma cloud described in the solar wind reference frame (left) and comet reference frame (right). The light grey circle indicates the boundary of the plasma cloud, the central point indicates the direction of the magnetic field off the page. The black horizontal arrows indicate the direction of the plasma cloud velocity in the solar wind reference frame $\boldsymbol{V}_{\mathrm{i}}$ and the solar wind velocity in the comet reference frame $\boldsymbol{V}_{\mathrm{SW}}$. The arrow denoted $\boldsymbol{E}_{\text {in }} \times \boldsymbol{B}$ indicates the direction of the drift of the electrons due to the polarisation electric field shown with a blue arrow. In the right-hand figure we also show the solar wind electric field in the comet reference frame $\left(E_{\mathrm{SW}}\right)$ and the net electric field (assuming in the figure that the magnitude of the solar wind and polarisation electric fields are the same). Angle $\alpha$ is the angle from the anti-sunward direction.

$\frac{\mathrm{d} \rho}{\mathrm{d} t}=\frac{2 \cdot 2 E_{\mathrm{in}}}{\mu_{0} V_{\mathrm{A}}}$

We thus get yet another contribution to the surface charge and the internal electric field, obtained analogously to the previous terms,

$\left(\frac{\mathrm{d} \boldsymbol{E}_{\text {in }}}{\mathrm{d} t} L_{\|}\right)_{3}=-\frac{1}{\varepsilon_{0}} \frac{2}{\mu_{0} V_{\mathrm{A}}} \boldsymbol{E}_{\mathrm{in}}$.

We illustrate the geometry of velocities and electric fields discussed above in Fig. 1. The angle $\alpha$ is the angle from the antisunward direction as defined later. In the right-hand figure we also show the direction of the solar wind electric field in the comet reference frame $\left(E_{\mathrm{SW}}\right)$, which occurs according to the Lorentz transform, as well as the direction of the net electric field.

For the situation at the comet we are interested in a quasisteady state situation, so we set the total rate of change of the electric field to 0 . A solution can then be found, which is written as

$\boldsymbol{E}_{\text {in }}=-\boldsymbol{V}_{i} \frac{K B}{1+K^{2}}-\boldsymbol{V}_{i} \times \boldsymbol{B} \frac{K^{2}}{1+K^{2}}$,

where $K$ is a dimensionless parameter defined as

$K=\frac{n_{\mathrm{C}} e \mu_{0} V_{\mathrm{A}} L_{\|}}{4 B}$.

The angle to the $-\boldsymbol{V}_{\boldsymbol{i}}$ direction of the electric field obtained through Eq. (9) is

$\alpha=\arctan (K)$

and the magnitude is

$\left|E_{\text {in }}\right|=V_{i} B \frac{K}{\sqrt{1+K^{2}}}$

where one may note that $V_{i} B$ is approximately the magnitude of the solar wind electric field in our application, as we assumed 

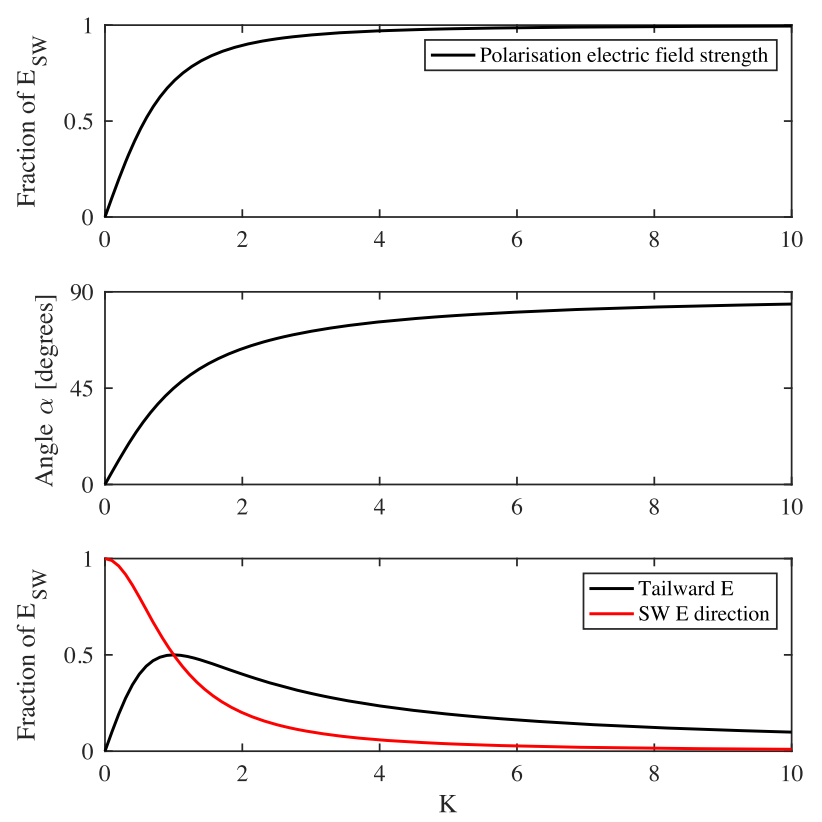

Fig. 2. Properties of the cloud model electric field as a function of the dimensionless parameter $K$ described in the text. Upper panel: magnitude of the cloud internal electric field as a fraction of the external (undisturbed) solar wind electric field. Middle panel: angle of the cloud electric field from the $-\boldsymbol{V}_{\mathbf{i}}$ direction. Lower panel: net electric field in the comet reference frame along the solar wind electric field direction (red line) and in the anti-sunward direction (black line) as a fraction of the undisturbed solar wind electric field.

that the magnetic field was not affected by the presence of the cloud. Should the local electron plasma be slowed down and the magnetic field correspondingly enhanced, we can thus replace $V_{i} B$ with an assumed local solar wind electric field of the surrounding undisturbed solar wind, as long as we can assume steady state and no field-aligned potential drops.

Figure 2 shows the magnitude of $E_{\text {in }}$ as a fraction of the solar wind electric field (Eq. (12)) as a function of K (upper panel) and the angle alpha given by Eq. (11) as a function of $\mathrm{K}$ in the middle panel. As can be seen for values of $\mathrm{K}$ above 10 the plasma cloud electric field is close to the solar wind electric field in magnitude, but an angular difference remains. When the polarisation electric field is close to that of the solar wind electric field in magnitude and nearly oppositely directed $\left(\alpha \approx 90^{\circ}\right)$ the solar wind electric field is approximately cancelled by the polarisation electric field, or in other words the plasma cloud is shielded from the solar wind electric field.

Our calculations so far have been made in the solar wind reference frame, where we have treated the cometary ions as a fast moving plasma cloud to show how the situation has many similarities to that of barium release experiments and other laboratory plasma cloud experiments. It is in practice more convenient to study the comet ionosphere in the comet reference frame. The only difference is that we should then add the undisturbed solar wind electric field to the above equations in agreement with the Lorentz transformation of the fields. In Fig. 2 the fraction of the solar wind electric field seen in the original solar wind electric field direction $\left(1-\left|E_{\mathrm{in}}\right| /\left|E_{\mathrm{SW}}\right| \sin (\alpha)\right)$ is shown with a red line and the anti-sunward electric field resulting from the angular difference between the solar wind and plasma cloud electric fields $\left(\left|E_{\text {in }}\right| /\left|E_{\mathrm{SW}}\right| \cos (\alpha)\right)$ is shown as a black line (lower panel). Both are shown as a fraction of the undisturbed solar wind electric field.

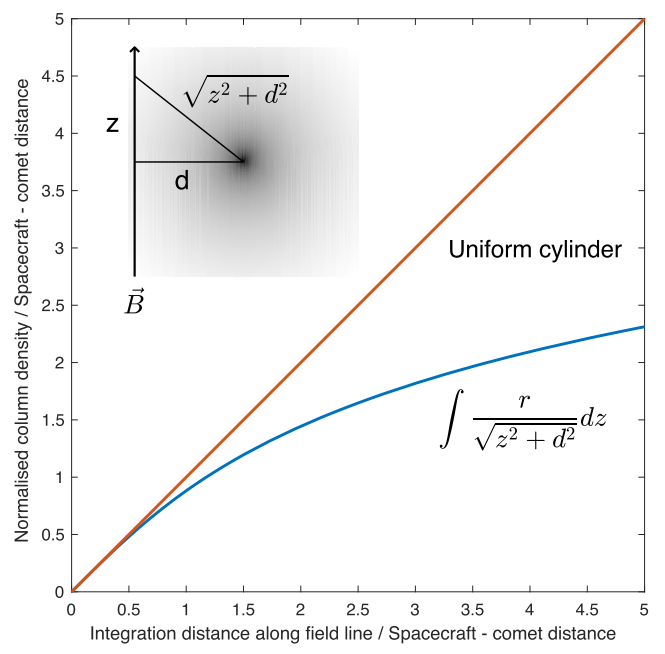

Fig. 3. Column density along a field line at the border of a plasma cloud for a uniform cylinder (red line) and for an ionosphere where the density falls off as $1 / r$ (blue line). The insert in the upper left corner illustrates the geometry of the $1 / r$ case.

\section{Application of the cloud model to a comet}

\subsection{Density profile of a comet}

A comet ionosphere is at a first glance not a uniform cylinder. The density close to the nucleus falls off as $1 / r$ (Edberg et al. 2015; Nilsson et al. 2015a), while further away on the dayside it may be assumed to fall off as $1 / r^{2}$ (Behar et al. 2018). However, the quantity of interest is not the local plasma density, but the density integrated along the field line. That parameter determines how much charge is accumulated on the field line at the edge of the cylinder. For a cylinder this becomes $n_{\mathrm{C}} L_{\|}$as used above. For a straight field line with closest approach to the nucleus at distance $d$, and a density $n(d) \propto 1 / r$ the integrated density along the field line becomes

$$
N(z, d)=n(d) \int \frac{d}{\sqrt{z^{2}+d^{2}}} \mathrm{dz}
$$

where $z$ is the distance along the field line and the spherical radius is $r=\sqrt{z^{2}+d^{2}}$; see Fig. 3 for an illustration. Integration of Eq. (13) yields the column density integrated out to a distance $z$ along the field line

$N(z, d)=n(d) d \ln \left(\frac{\sqrt{z^{2}+d^{2}}+z}{\sqrt{z^{2}+d^{2}}-z}\right)$,

where $N$ is the column density integrated from 0 towards positive $z$. We can see that the integral does not vanish at infinity, we must thus stop the integration at some point. The reason is that the description of the density falling off as $1 / r$ is not valid at large distances. One may note that the integrated density is proportional to $d$ and to the logarithm of the distance over which we perform the integration, so it will increase only slowly with $z$. Inspection of Fig. 3 shows that integration over a distance $z$ corresponding to a few $d$ ( $x$-axis of Fig. 3 ) yields a value corresponding to a uniform cylinder of length of about $2 r$ ( $y$-axis of Fig. 3. Figure 3 shows the integral from 0 to $z$ so it should be multiplied by 2 to obtain the total value including also negative $z$, obtaining a value up to about 4 .

Above we have shown that the integrated column density in the region where the plasma density falls off as $1 / r$ is roughly 
proportional to $d n(d)$. As $n(d) \propto 1 / d$ this means that the column density is approximately constant. Thus the constant density cylinder is in a sense a rather good model for the comet ionosphere.

As long as the comet ionosphere density falls off as $1 / r$, we are inside the cylinder, and no charge accumulation arises. The ionospheric density falls off with the rate $1 / r$ because the background neutral atmosphere is constantly ionised at a slow rate, adding plasma to the ionosphere as it expands into space. This breaks down once most of the atmosphere has been ionised. This is not relevant because it takes 10-100 days before this happens with an ionisation rate of the order of $10^{-7}$ to $10^{-6} \mathrm{~s}^{-1}$ (Galand et al. 2016). The $1 / r$ dependence rather breaks down owing to the acceleration of the ions (Behar et al. 2018). It is known from observations that most of the ions at energies observable by the RPC-ICA instrument are moving anti-sunward (Nilsson et al. 2017, 2015a; Behar et al. 2016; Berčič et al. 2018). At some point on the front of the comet ionosphere the density must thus drop much faster than $1 / r$. This may in practice happen because of the polarisation electric field, which has an anti-sunward component such that the initial boundary formation may be self-sustaining.

The cloud model predicts a constant electric field inside the cloud. Let us consider the case of a constant uniform electric field $\boldsymbol{E}$ pointing opposite to the neutral gas expansion velocity of a comet. Let us also assume that the neutral gas velocity is negligible compared to the velocity the ions soon acquire through acceleration in the electric field. Newly produced ions start to move along the electric field and get gradually higher energy. For an observer somewhere downstream along this electric field, the energy scale is also a distance scale. The further away the ions were produced, the higher their energy. We can thus assume that all particles measured in some energy range were added in some sampling volume at some particular distance. The flux of particles from a sampled volume is then the flux of newly produced ions from that volume, i.e. the production rate integrated over the observation region. This also remains true if the magnetic field is of some significance, perturbing the straight line. As long as the trajectory is only a part of a full gyration it is true that ions with higher energy were born further away.

If we assume that all ions move straight along the electric field direction, then the production rate is related to the differential particle flux measured downstream as the total flux from the sampled volume divided by the depth $\Delta r$ of the sampled volume

$q=\frac{j \Delta W \Delta \Omega}{\Delta r}$

where $j$ is the differential flux of particles in the sampled volume, $\Delta W$ the energy resolution of the instrument in $\mathrm{eV}$, and $\Delta \Omega$ the solid angle of the instrument field of view. The value $\Delta r$ is related to the energy resolution of the instrument $\Delta W[\mathrm{eV}]$ and the magnitude of the electric field as $\Delta r=\Delta W[\mathrm{eV}] / E$. We therefore need to know or assume the magnitude of $E$ in order to calculate the production rate $q$. If we instead settle for an approximation of the density, this can be obtained by multiplying with $\Delta r$ to obtain the total production in the source region, which is the flux out of the source region, and divide with the ion velocity

$n=\frac{j \Delta W \Delta \Omega}{\Delta r} \frac{\Delta r}{v}=\frac{j \Delta W \Delta \Omega}{v}$,

such that we can get an estimate of the density produced in the source region without actually knowing the electric field. If we

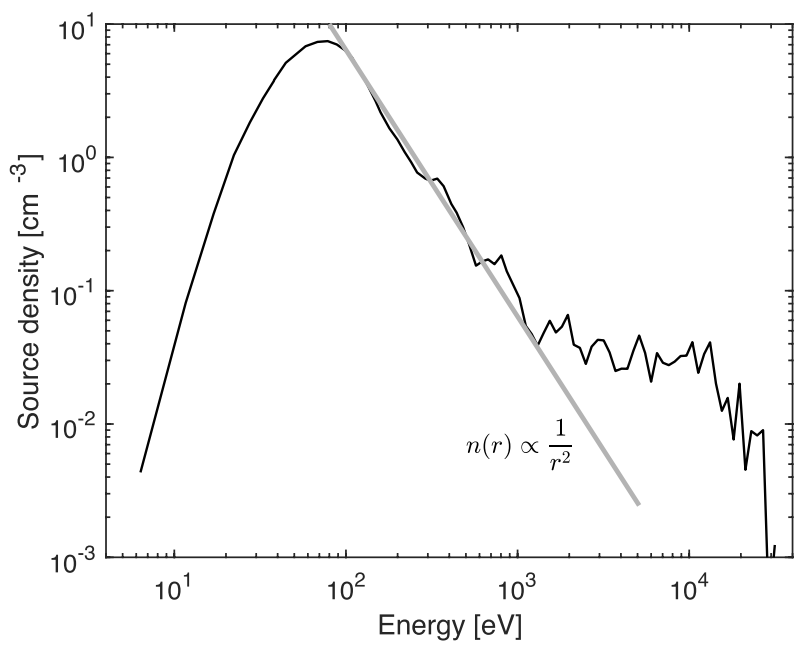

Fig. 4. Estimated density in the source region $\left[\mathrm{cm}^{-3}\right]$ as a function of energy $[\mathrm{eV}]$, which is also a distance scale. A thick grey line corresponds to a slope $n_{\text {source }} \propto 1 / r^{2}$.

set the velocity to that of the neutral gas we get the flux of just ionised particles in the volume. The result of Eq. (16) is actually rather obvious, it is the density corresponding to the flux out of the sampling volume when we assume the velocity to be that of the neutral gas. This is an estimate of the density of the locally produced ions before they experience significant acceleration, built up over the total column depth $\Delta r$ of the sampling volume.

If the ionisation rate is constant, the production rate and ion density in the source region are both proportional to the neutral atmosphere density in the sampled volume. The energy spectra of an ion spectrometer can thus be used to get an estimate of the neutral gas density profile in the upstream region. In Fig. 4 we show a sample energy spectrum of water ions, obtained using the RPC-ICA mass resolving ion spectrometer on board Rosetta (Nilsson et al. 2007). We have chosen to use data from 16 June 2015, a day when ICA detected water ions up to energies of about $20 \mathrm{keV}$. Rosetta was then located at a distance of $220 \mathrm{~km}$ from the comet nucleus and at 1.4 au from the Sun. The inferred ion density of the source region, assuming a neutral gas velocity of $1 \mathrm{~km} \mathrm{~s}^{-1}$, is shown as a solid black line. A thick grey line shows the slope corresponding to a $1 / r^{2}$ dependence and a constant uniform electric field. From about $100 \mathrm{eV}$ and up to an energy of about $1 \mathrm{keV}$ we see that the inferred density at the source follows an $1 / r^{2}$ dependence. This is what is expected for the neutral gas density, and with constant production of ions for the ion density as well. We can therefore say that we have an observation that is consistent with the ion density falling off as $1 / r^{2}$ and the column density is thus no longer constant. Typically the $1 / r^{2}$ dependence is not present at energies above $1 \mathrm{keV}$; we here show an example with significant flux above $1 \mathrm{keV}$.

\subsection{Currents flowing in a comet ionosphere}

The value of the model lies in that we obtain a simple analytical estimate of the polarisation electric field as a function of the currents flowing in and around the ionosphere. The important factor determining the polarisation electric field is the balance between currents in the enhanced plasma density region giving rise to charge accumulation, and field-aligned currents removing the excess charge at the outer boundary. The field-aligned 
currents are described as arising because of a divergence of the perpendicular electric field at the boundary. This coupling is not strongly affected if the coupling instead occurs over a larger area owing to a gradually decreasing plasma cloud density. Thus the fact that we do not know where and precisely how the integrated column density of the comet ionosphere drops to background levels does not strongly affect our estimates of the polarisation electric field.

The polarisation electric field is determined from in situ estimates of the currents flowing in the plasma cloud interior, i.e. the ion current and the estimate of the $\boldsymbol{E} \times \boldsymbol{B}$ drift of the electrons. If the field-aligned coupling of our current system to the solar wind occurs over an extended distance this affects the shape of the internal electric field of the plasma cloud. The field is constant inside the charge accumulation region and behaves like a line dipole electric field outside. If the charge accumulation region is spatially extended, we get a mixture of inside and outside electric fields. We investigate this effect in Appendix A, where we look at the electric field obtained when ion and electron distributions falling off as $1 / r$ outside some inner region are displaced just so much that a reasonable electric field is produced. We find that in such a case the equipotential lines inside the plasma cloud are no longer straight lines; they emanate from a central region. In terms of electric fields, the electric field inside the plasma cloud curve around the central part. This affects the precise electric field directions, but not the general features.

Another potential problem with applying the cloud model to a comet is that the magnetic field is not undisturbed by the presence of the plasma, as could be assumed in the work of Brenning et al. (1991). The magnetic field in the comet environment is enhanced as compared to the surrounding solar wind (Goetz et al. 2017). Whereas the magnetic field in the vicinity of the comet was very variable, data from the dayside excursion indicated a rather constant magnetic field within the comet magnetosphere (Goetz et al. 2017; Edberg et al. 2016). The role of the magnetic field in our simple model is to determine the current carried by the $\boldsymbol{E} \times \boldsymbol{B}$ drift of the electrons in the solar wind reference frame. The locally observed magnetic field should thus serve us well to give an estimate of this current. The magnetic field also occurs in our equation for the field-aligned currents, through the dependence of the Alfvén velocity on the magnetic field. The Alfvén velocity of the surrounding plasma can be assumed to be that for the undisturbed solar wind.

We also note that in our very simple model, by assuming steady state we do not take into account slowing down of the cometary ions in the solar wind reference frame. The upper energy we observe, about $1 \mathrm{keV}$, corresponds to a velocity of about $100 \mathrm{~km} \mathrm{~s}^{-1}$ for water ions in the comet reference frame and a corresponding slowing down in the solar wind reference frame. This would correspond to a weakening of the ion current in our model of about $25 \%$ (assuming a solar wind velocity of $400 \mathrm{~km} \mathrm{~s}^{-1}$ ), if the entire comet ionosphere moved with this velocity. Most of the comet ionosphere move at a much slower velocity. Thus this should not strongly affect our results.

The field-aligned currents connect the plasma cloud to surrounding space, forming Alvén wings. On a larger scale this leads to field-line draping. This is not part of our simple model.

\section{Polarisation electric field of comet 67P}

To apply the cloud model to the situation at the comet we must determine suitable values for the drift velocity of the cloud ions
$V_{i}$, the magnetic field $B$, the Alfvén velocity of the surrounding medium, and a column plasma density $n_{\mathrm{C}} L_{\|}$.

All the above parameters vary throughout the mission. By inspecting Eq. (10) and using the formula for the Alfvén velocity we see that

$K \propto \frac{n_{\mathrm{C}} L_{\|}}{\sqrt{n_{\mathrm{SW}}}} \frac{B_{\mathrm{SW}}}{B}$,

where we can note that $\sqrt{n_{\mathrm{SW}}}$ vary much less than the comet ion density throughout the mission, so we can to a first approximation fix the solar wind density value to something intermediate, say $3 \mathrm{~cm}^{-3}$. The other factor affecting $K$ is the ratio of the solar wind to comet magnetic field strength. The local magnetic field is measured by the magnetometer instrument (MAG) of the Rosetta orbiter (Glassmeier et al. 2007). The magnetic field of the surrounding solar wind can be estimated from a simple Parker model (Parker 1958), where we assumed an average magnetic field at Earth of $6.8 \mathrm{nT}$ and an average Parker spiral angle at Earth of $45^{\circ}$. The radial component is assumed to fall off as $1 / R^{2}$ and the azimuthal component is assumed to fall off as $1 / R$, where $R$ is the distance to the Sun.

The local plasma density is measured by the mutual impedance probe (MIP; Trotignon et al. 2007) and the langmuir probe (LAP; Eriksson et al. 2007) instruments on board Rosetta. We can turn this into an approximate column density, corresponding to $n_{\mathrm{C}} L_{\|}$in the equations above, by multiplying by the distance to the nucleus at the observation point and some factor. From Eq. (14) and Fig. 3 we see that a reasonable choice for this factor is up to about 4 , as one would have to use very high values of $z$ (integration along the field line) to get higher values. We have therefore used the value 4 .

With these assumptions in place, we can now plot one of the main parameters, the anti-sunward electric field as a fraction of $V_{i} B$ (essentially the solar wind electric field) as a function of the cometary ion column density $\left(n_{\mathrm{C}} L_{\|}\right)$and the solar wind to comet magnetic field strength ratio $\left(B_{\mathrm{SW}} / B\right)$, as shown in Fig. 5 On top of this the distribution of data points from the MIP and MAG instruments that fall into the corresponding parameter space is shown as contour levels of $\log _{10}$ of the number of data points. We used a list of MIP density points throughout the mission and interpolated MAG magnetic field data to the same times. Using instead density estimates from the LAP instrument yields the same general picture (which instrument provides the best density estimate depends on conditions, but the details are not important here).

As can be seen we indeed find that most of the observations at the comet fall in a regime where the tailward electric field of the comet is significant. This is however not the full story. We saw from Fig. 2 that for values of the parameter $K$ around unity, we may have a significant polarisation electric field directed at around $45^{\circ}$ from the anti-sunward direction. Indeed a significant part of the observations at comet $67 \mathrm{P}$ fall in this category, as shown in Fig. 6.

For low column densities we are in a regime where the antisunward electric field is small, the magnitude of the plasma cloud electric field in total is small, and the ions follow the cycloid trajectories expected for test particles. For high column densities the solar wind is more efficiently shielded and the tailward electric field also vanishes within the comet ionosphere. Most of the observations obtained at comet 67P were obtained in a regime between these two extremes. We investigate this further by plotting the model value of the net electric field, polarisation plus solar wind electric field, in the vicinity of comet $67 \mathrm{P}$ as a 


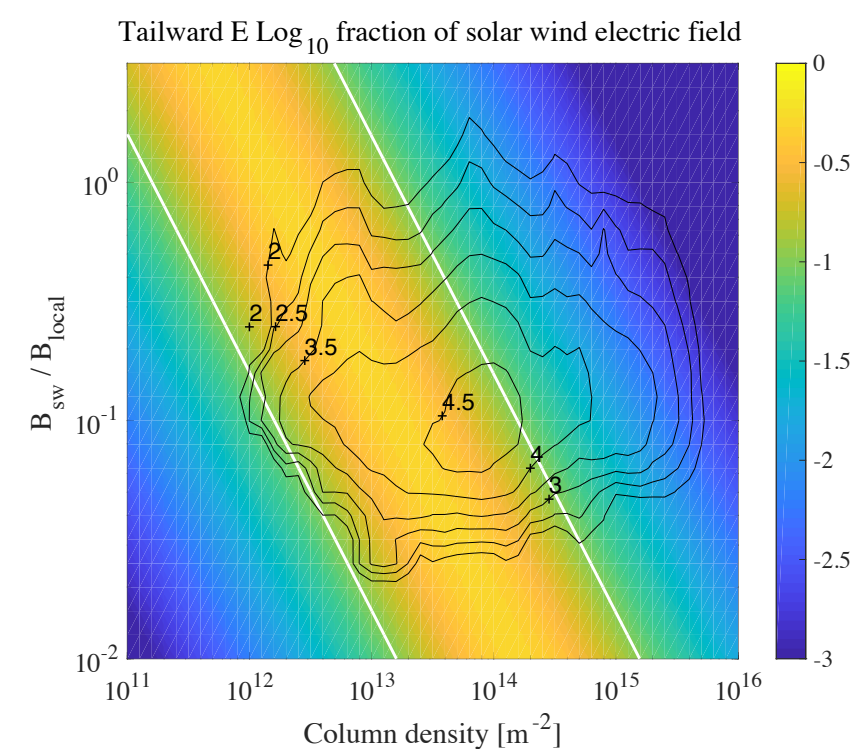

Fig. 5. Tailward polarisation electric field as a $\log _{10}$ fraction of the solar wind electric field (colour scale) as a function of the column ion density $\left[\mathrm{m}^{-2}\right]$ and the ratio of the solar wind to comet magnetic field strength. Black contour lines indicate the $\log _{10}$ number of data points to fall into the corresponding parameter space. We use density estimates obtained by the MIP instrument. White lines indicate the limits where the tailward electric field is $10 \%$ of the solar wind electric field.

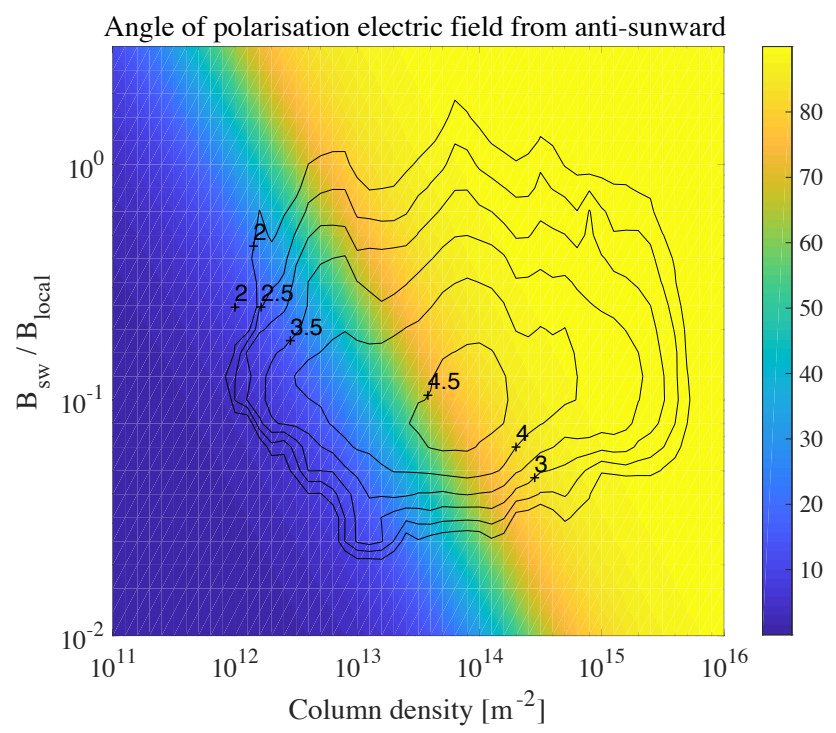

Fig. 6. Angle of the polarisation electric field from anti-sunward (colour scale) as a function of the column ion density $\left[\mathrm{m}^{-2}\right]$ and the ratio of the solar wind to comet magnetic field strength. Black contour lines indicate the $\log _{10}$ number of data points to fall into the corresponding parameter space.

function of time in Fig. 7. The MIP density data set we used consists of $3.7 \times 10^{6}$ data points. We note that the MIP density estimates from the dayside and nightside excursions (seen clearly in the cometocentric distance plot) were sparse and not reliable since the in situ plasma density was too low for the instrument measurements capabilities. We leave a closer examination of the excursion results and other low density regions to a future study.

Near perihelion, when the comet ionosphere was at its largest and densest, the net electric field was small and anti-sunward. Away from perihelion, the net electric field was typically a few
$0.1 \mathrm{mV} \mathrm{m}^{-1}$. At low activity the net electric field nearly reaches solar wind levels at about $1 \mathrm{mV} \mathrm{m}^{-1}$, but with a direction typically a few $10^{\circ}$ away from the undisturbed solar wind electric field direction $\left(\right.$ at $90^{\circ}$ ). The magnitude of the net electric field indicates that the observed anti-sunward streaming cometary ions with a typical energy range of about $100-1000 \mathrm{eV}$, must have been accelerated over distances of 100-10000 km, for electric fields in the range 0.1 and $1 \mathrm{mV} \mathrm{m}^{-1}$. The spectra shown in Fig. 4 was obtained in June 2015 when the net model electric field was about $0.25 \mathrm{mV} \mathrm{m}^{-1}$. The outer limit of the region where the inferred density fell off as $1 / r^{2}$ thus corresponds to a distance of about $4000 \mathrm{~km}$ (at an energy of about $1 \mathrm{keV}$ ). Outside of that region the fluxes of ions fell off with a lower rate with energy/inferred distance. As it is not likely that there was an increased source of ions at that distance, it is more likely that the source was a region of more intense heating of the ions and that the uniform electric field approximation breaks down. This could for example correspond to a cometary ion shock, which may thus be remotely probed by the energy spectra of observed cometary ions.

\section{Comparison with observations}

We leave a closer comparison with observed flow directions for a future study. The real situation is complicated by the fact that the solar wind is deflected by mass loading (Behar et al. 2017), although this can likely be regarded as a gyration in the slowed down plasma flow inside the comet ionosphere (Behar et al. 2018). The electric field of the deflected solar wind could also contribute to a tailward acceleration of cometary ions, which our current model does not take into account. It is still worthwhile to compare with a few published studies of cometary ion flow.

Nilsson et al. (2015b) discussed the first observations obtained as Rosetta approached comet 67P. They showed an example from 21 September 2014, in which the cometary ion flow direction was close to $90^{\circ}$ from the proton flow direction. Our model result is not in agreement with this, a typical angle for that period was smaller, i.e. $50-70^{\circ}$. The model results for the early period from August and September 2014 appear in general to have an underestimate of the cone angle of the cometary flow as compared to observations. Behar et al. (2016) showed solar wind deflection and cometary ion flow data for a case on 28 November 2014, when activity had increased a bit. The average angle between the proton and cometary ion flow directions was $60^{\circ}$ and our model results are in the range of $50-60^{\circ}$ for that day.

Nilsson et al. (2017) showed that cometary ion energies initially increased with comet activity, but then decreased around perihelion, approximately between July and October 2015, when activity peaked. October was dominated by the dayside excursion, which showed more accelerated ions at larger distance from the comet. The local minimum in cometary ion energies around perihelion agrees with the minimum in the net electric field of our model.

Berčič et al. (2018) showed how the cometary ion data obtained in terminator orbits can typically be divided into two groups. One at lower energy moving radially away from the comet in the terminator plane, and the other moving along the solar wind electric field direction in the terminator plane. Both populations showed a dominating anti-sunward flow component, which is persistent in the Rosetta ion observations (Nilsson et al. 2017, 2015a). Thus the higher energy ions coming from further out were not completely shielded from the solar wind electric field. The inner population may have been somewhat influenced by the solar wind electric field, but an ambipolar electric field leading to the radial flow in the terminator plane 

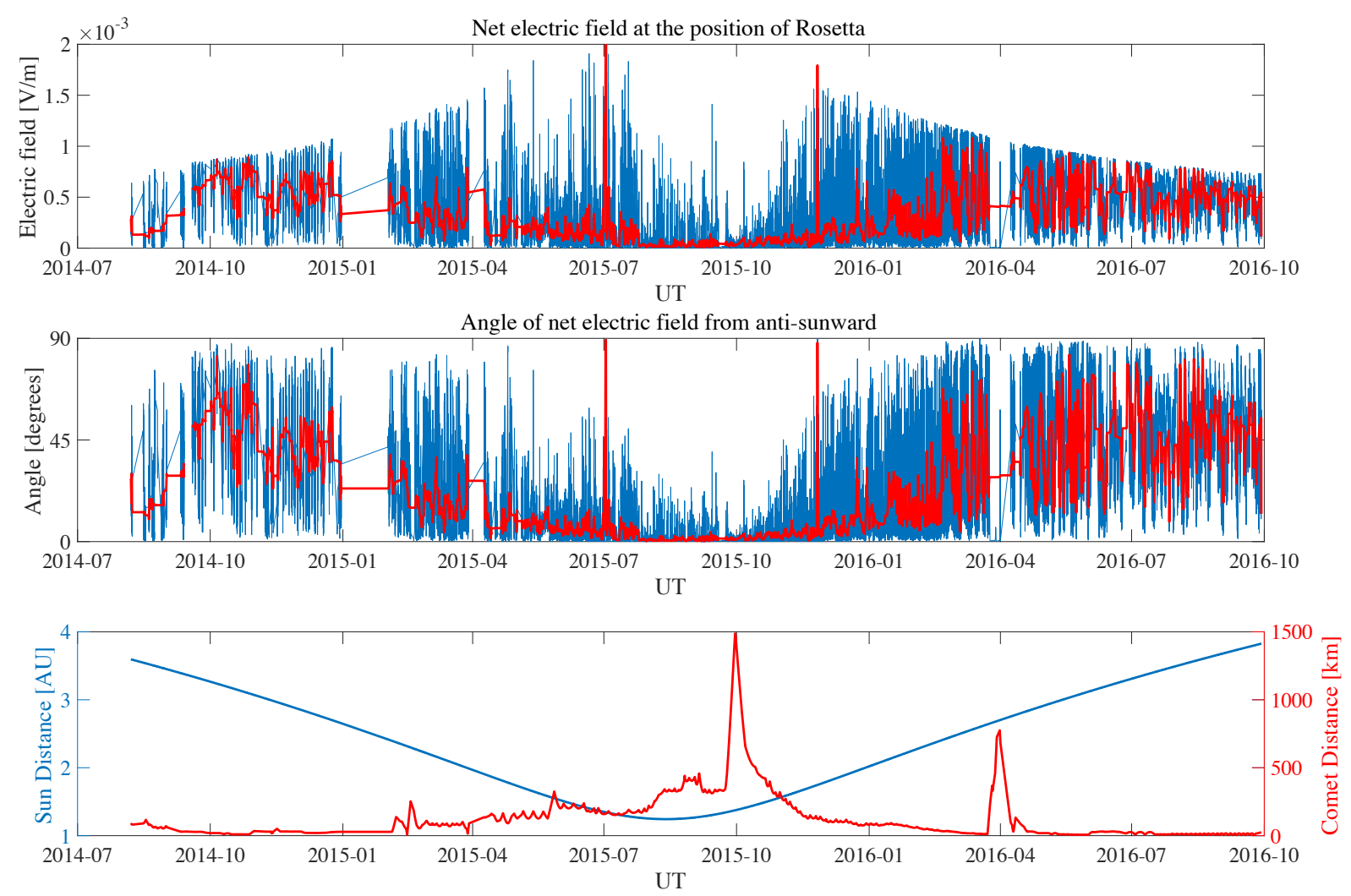

Fig. 7. Model electric field based on observations and an assumed solar wind electric field. Blue lines show the model results; red lines show a 1000 data points running mean of the same data. Upper panel: magnitude of the model electric field. Middle panel: angle of the net model electric field from anti-sunward. Lower panel: Sun distance [AU] as a blue line, scale to the left, cometocentric distance [km] as a red line, scale to the right.

appeared to dominate. Such an ambipolar electric field and its effect on cometary ions have been modelled by Vigren \& Eriksson (2017). These results indicate that the electric field of the magnetosphere and ionosphere of the comet is not as homogeneous as assumed in our model and that for the full picture we need to take into account the ambipolar electric field of the expanding ionosphere present at close distance to the nucleus.

A shielding of the inner part of the comet ionosphere is in general agreement with the deflection of solar wind ions reported in Behar et al. (2017). If solar wind ions enter the plasma cloud with its local electric field environment, there is no longer a balance between the electric field and the $-\boldsymbol{v} \times \boldsymbol{B}$ force acting on the ions. As shown by Behar et al. (2018) such a gyration also occurs in a simple mass loading model. The increased mass loading leads to a slower flow of the electron plasma, which has similarities to the polarisation electric field arising because of the slow moving cometary ions. The mass-loading model used a realistic cometary ion density profile and should be more suitable to study solar wind ion trajectories in the mass-loaded plasma at larger scales, and also makes a predictions on the direction of the electric field. The plasma cloud model makes a prediction on the direction of the local electric field close to the nucleus and how the cometary plasma connects to surrounding space in terms of field-aligned currents. We leave a closer comparison of these two models for a future study.

\section{Conclusions}

We have found that the important parameters (plasma density, ion velocity, and magnetic field strength) at comet $67 \mathrm{P}$ are in the right range for a significant polarisation electric field to build up. Such a field has some important consequences observed in data.

(1) Once activity rises enough, the electric field of the comet ionosphere to a large extent cancels the solar wind electric field inside the comet ionosphere. There the ambipolar electric field likely dominates.

(2) The cancellation is not perfect, which results in a significant anti-sunward directed electric field with a strength of the order of $10 \%$ of the magnitude of the solar wind electric field.

(3) There is also an intermediate region in which the resulting electric field has an anti-sunward component but an angle compared to the anti-sunward direction in the range $0-90^{\circ}$. This often corresponds to the situation at comet $67 \mathrm{P}$ during the Rosetta mission.

Point (1) above also has consequences for the solar wind ions that gyrate in the local magnetic field once they are inside the comet ionosphere, as there is no longer a balance between the electric field and the $-\boldsymbol{v} \times \boldsymbol{B}$ force acting on the ions. This however likely also happens further out in a mass-loaded plasma (Behar et al. 2018).

An anti-sunward directed electric field was postulated to exist based on observations of anti-sunward streaming cometary ions throughout most of the Rosetta mission as reported in Nilsson et al. (2015a, 2017), Behar et al. (2016), Berčič et al. (2018). The observations appear to fit rather well to our simple model, but a somewhat more sophisticated model should be used to fully assess if we can explain the actually observed ion energies and flow directions. The upper energy of cometary ions observed were mostly in the range $100 \mathrm{eV}$ to $1 \mathrm{keV}$. This 
can be compared with an estimate of the tailward electric field of about $0.1-1 \mathrm{mV} \mathrm{m}^{-1}$ yielding acceleration over distances of $100-10000 \mathrm{~km}$.

That the observed energy spectra in our example fall off as $1 / r^{2}$ supports the existence of a large-scale anti-sunward directed electric field. If one instead assumes that the ions are $\boldsymbol{E} \times \boldsymbol{B}$ drifting within a slowed down solar wind, then they would not be continually accelerated and would show up as a drifting cold population.

We conclude that the simple cloud model we used can point towards the relevant physics involved in the interaction between a small comet ionosphere and the solar wind and provide some relevant quantitative estimates of the electric field strength and direction in the vicinity of the comet. For more detailed studies a proper simulation model is still needed, and our results indicate that important physics will be missed unless electrons and charge accumulation are taken properly into account.

A final note is that as the cometary ions are moving mostly anti-sunward, not following the cycloid trajectories, which would have resulted without the polarisation electric field, one can say that the net effect of the polarisation electric field is to provide a more fluid-like behaviour also at small scales for the cometary ions. It may be possible to describe the major ions with a fluid model; a generalised Ohm's law accounts for the ion currents along the electric field at small scales. The appearance of a solar wind cavity for a small-scale comet (Behar et al. 2017; Nilsson et al. 2017), on the other hand, appears to be an effect where the gyroradius of the solar wind ions is important. Thus the main ion species behave fluid-like and for the minor species the gyroradius is important both in the solar wind and the comet ionosphere. Once solar wind ions enter a shielded comet ionosphere smaller than an ion inertial length, the ions gyrate. The bulk motion is thus turned into something acting as a thermal motion of the plasma just as at a shock. The same is also true for a gradual decrease of the electric field and associated slow down of the electron fluid (Behar et al. 2018).

Acknowledgements. Rosetta is a European Space Agency (ESA) mission with contributions from its member states and the National Aeronautics and Space Administration (NASA). The work on RPC-ICA was funded by the Swedish National Space Board under contracts 108/12,112/13, 96/15, 94/11 and by the Swedish Research Council under contract 2015-04187. This work benefitted from the International Space Science Institute (ISSI) support for project 2017402, Plasma Environment of Comet 67P after Rosetta. Work at the Royal Belgian Institute for Space Aeronomy was supported by the Belgian Science Policy Office through the Solar-Terrestrial Centre of Excellence and by PRODE $x$ /ROSETTA/ROSINA PEA 4000107705. Work at Umeå University was funded by SNSB grant 201/15. Work at LPC2E/CNRS was supported by ESEP, CNES, and by ANR under the financial agreement ANR-15-CE31-0009-01. The RPC-ICA data is available through the PSA archive of ESA. We are indebted to the whole Rosetta mission team, Science Ground Segment, and Rosetta Mission Operation Control for their hard work making this mission possible.

\section{References}

Alfvén, H. 1957, Tellus, IX, 92

Behar, E., Nilsson, H., Wieser, G. S., et al. 2016, Geophys. Res. Lett., 43, 1411 Behar, E., Nilsson, H., Alho, M., Goetz, C., C., \& Tsurutani, B. 2017, MNRAS, 469, 5396

Behar, E., Tabone, B., Saillenfest, M., et al. 2018, A\&A, DOI: 10.1051/0004-6361/201832736

Berčič, L., Behar, E., Nilsson, H., et al. 2018, A\&A, 613, A57

Bieler, A., Altwegg, K., Balsiger, H., et al. 2015, A\&A, 583, A7

Biver, N., Hofstadter, M., Gulkis, S., et al. 2015, A\&A, 583, A3

Brenning, N., Kelley, M. C., Providakes, J., Stenbaek-Nielsen, H. C., \& Swenson, C. 1991, J. Geophys. Res., 96, 9735

Carr, C., Cupido, E., Lee, C. G. Y., et al. 2007, Space Sci. Rev., 128, 629

Coates, A. J., Burch, J. L., Goldstein, R., et al. 2015, J. Phys. Conf. Ser., 642, 012005

Cravens, T. \& Gombosi, T. 2004, Adv. Space Res., 33, 1968

Damas, M. C., Flammer, K. R., \& Mendis, D. A. 1994, ApJ, 421, 355

Deca, J., Divin, A., Henri, P., et al. 2017, Phys. Rev. Lett. 118, 205101

Dubinin, E., Lundin, R., Norberg, O., \& Pissarenko, N. 1993, J. Geophys. Res., 98, 3991

Edberg, N. J. T., Eriksson, A. I., Odelstad, E., et al. 2015, Geophys. Res. Lett., 42,4263

Edberg, N. J. T., Alho, M., André, M., et al. 2016, MNRAS, 462, S45

Eriksson, A. I., Boström, R., Gill, R., et al. 2007, Space Sci. Rev., 128, 729

Eriksson, A. I., Engelhardt, I. A. D., André, M., et al. 2017, A\&A, 605, A15

Galand, M., Héritier, K. L., Odelstad, E., et al. 2016, MNRAS, 462, S331

Glassmeier, K.-H., Richter, I., Diedrich, A., et al. 2007, Space Sci. Rev., 128, 649

Goetz, C., Volwerk, M., Richter, I., \& Glassmeier, K.-H. 2017, MNRAS, 469, S268

Haerendel, G. 1982, Z. Naturforsch., 37, 728

Hässig, M., Altwegg, K., Balsiger, H., et al. 2015, Science, 347, 0276

Herlofson, N. 1951, Arkiv för fysik, 3, 247

Huang, Z., Tóth, G., Gombosi, T. I., et al. 2018, MNRAS, 475, 2835

Koenders, C., Goetz, C., Richter, I., Motschmann, U., \& Glassmeier, K.-H. 2016, MNRAS, 462, S235

Lee, S., von Allmen, P., Allen, M., et al. 2015, A\&A, 583, A5

Mallinckrodt, A. J. \& Carlson, C. W. 1978, J. Geophys. Res.: Space Phys., 83, 1426

Mandt, K. E., Eriksson, A., Edberg, N. J. T., et al. 2016, MNRAS, 462, S9

Neugebauer, M. 1990, Rev. Geophys., 28, 231

Nilsson, H., Lundin, R., Lundin, K., et al. 2007, Space Sci. Rev., 128, 671

Nilsson, H., Stenberg Wieser, G., Behar, E., et al. 2015a, A\&A, 583, A20

Nilsson, H., Stenberg Wieser, G., Behar, E., et al. 2015b, Science, 347, 0571

Nilsson, H., Stenberg Wieser, G., Behar, E., et al. 2017, MNRAS, 469, S252

Odelstad, E., Stenberg-Wieser, G., Wieser, M., et al. 2017, MNRAS, 469, S568

Ogino, T., Walker, R. J., \& Ashour-Abdalla, M. 1988, J. Geophys. Res.: Space Phys., 93, 9568

Parker, E. N. 1958, ApJ, 128, 664

Simon Wedlund, C., Alho, M., Gronoff, G., et al. 2017, A\&A, 604, A73

Tonks, L. 1931, Phys. Rev., 37, 1458

Trotignon, J. G., Michau, J. L., Lagoutte, D., et al. 2007, Space Sci. Rev., 128, 713

Vigren, E. \& Eriksson, A. 2017, AJ, 153, 150 


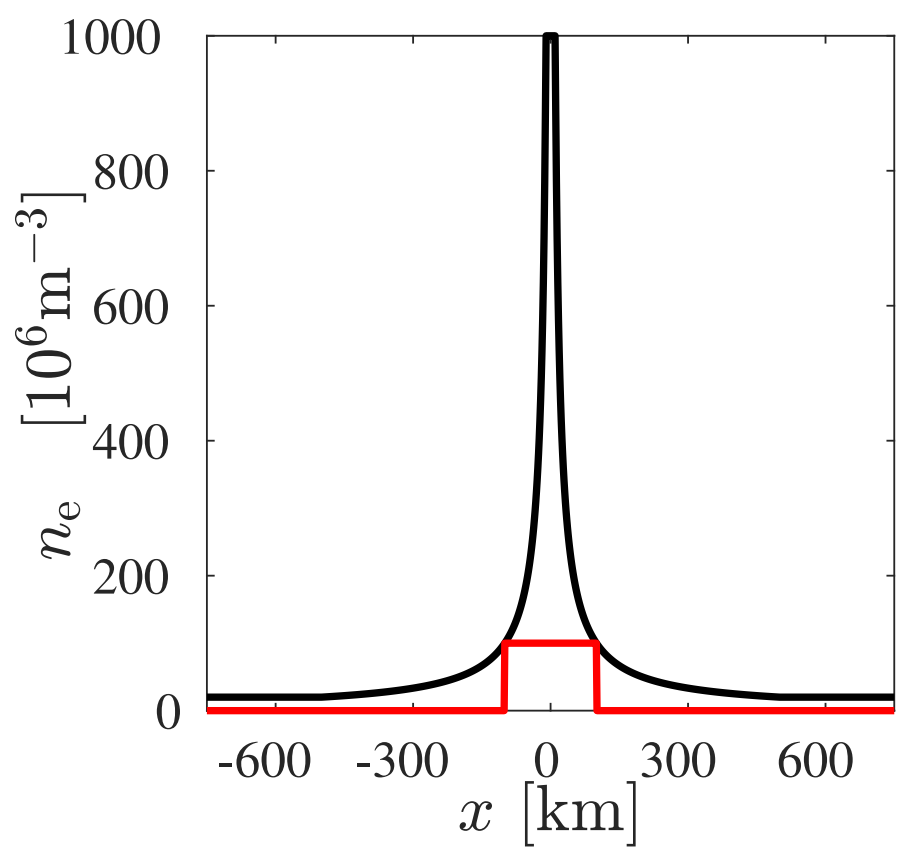

Fig. A.1. Plasma density along the $x$-axis in two different density models. The red curve shows a plasma with a uniform density $n_{0}$ within a cylinder of radius $R_{0}$, and the black curve shows a plasma with a density given by Eq. (A.3).

Table A.1. Parameters used in the examples shown in the figures in Appendix A.

\begin{tabular}{cc}
\hline \hline$n_{0}$ & $1.0 \times 10^{8} \mathrm{~m}^{-3}$ \\
$R_{0}$ & $100 \mathrm{~km}$ \\
$\Delta x$ & $5 \mathrm{~mm}$ \\
$b$ & $10 \mathrm{~km}$ \\
$d$ & $500 \mathrm{~km}$ \\
\hline
\end{tabular}

\section{Appendix A: Electric field}

In a uniform plasma bounded by sheaths, if the ions are displaced a distance $\Delta x$ with respect to the electrons, the resulting electric field is uniform inside the plasma and equal to (Tonks 1931)

$\boldsymbol{E}_{\mathbf{i}}=\left[-\frac{1}{N_{\mathrm{D}}} \frac{e \Delta x n_{0}}{\epsilon_{o}}, 0,0\right]$,

where $N_{\mathrm{D}}$ is the number of dimensions: $N_{\mathrm{D}}=1$ for planar, $N_{\mathrm{D}}=2$ for cylindrical, and $N_{\mathrm{D}}=3$ for spherical geometry. In cylindrical geometry and with a cylinder of radius $R_{0}$, the field outside this cylinder is (Herlofson 1951; Brenning et al. 1991)

$\boldsymbol{E}_{\mathbf{o}}=\frac{1}{2} \frac{\rho_{0}}{\varepsilon_{0}} R_{0}^{2}\left[\frac{x^{2}-y^{2}}{\left(x^{2}+y^{2}\right)^{2}}, \frac{2 x y}{\left(x^{2}+y^{2}\right)^{2}}, 0\right]$.

The density along the $x$-axis is shown by the red curve in Fig. A.1 for a plasma cylinder aligned with the $z$-axis, which has a circular cross section in the $x-y$ plane. The parameters are shown in Table A.1.

The electric field given by Eqs. (A.1) and (A.2) is shown in Fig. A.2, $E_{x}$ in panel a, $E_{y}$ in panel b, and panel c shows the plasma potential $V_{\mathrm{p}}$ obtained by numerically integrating the electric field.

The density profile for a cylinder with a $1 / r_{\mathrm{c}}$ density dependence between an inner boundary at radius $r_{\mathrm{c}}=b$ and an outer
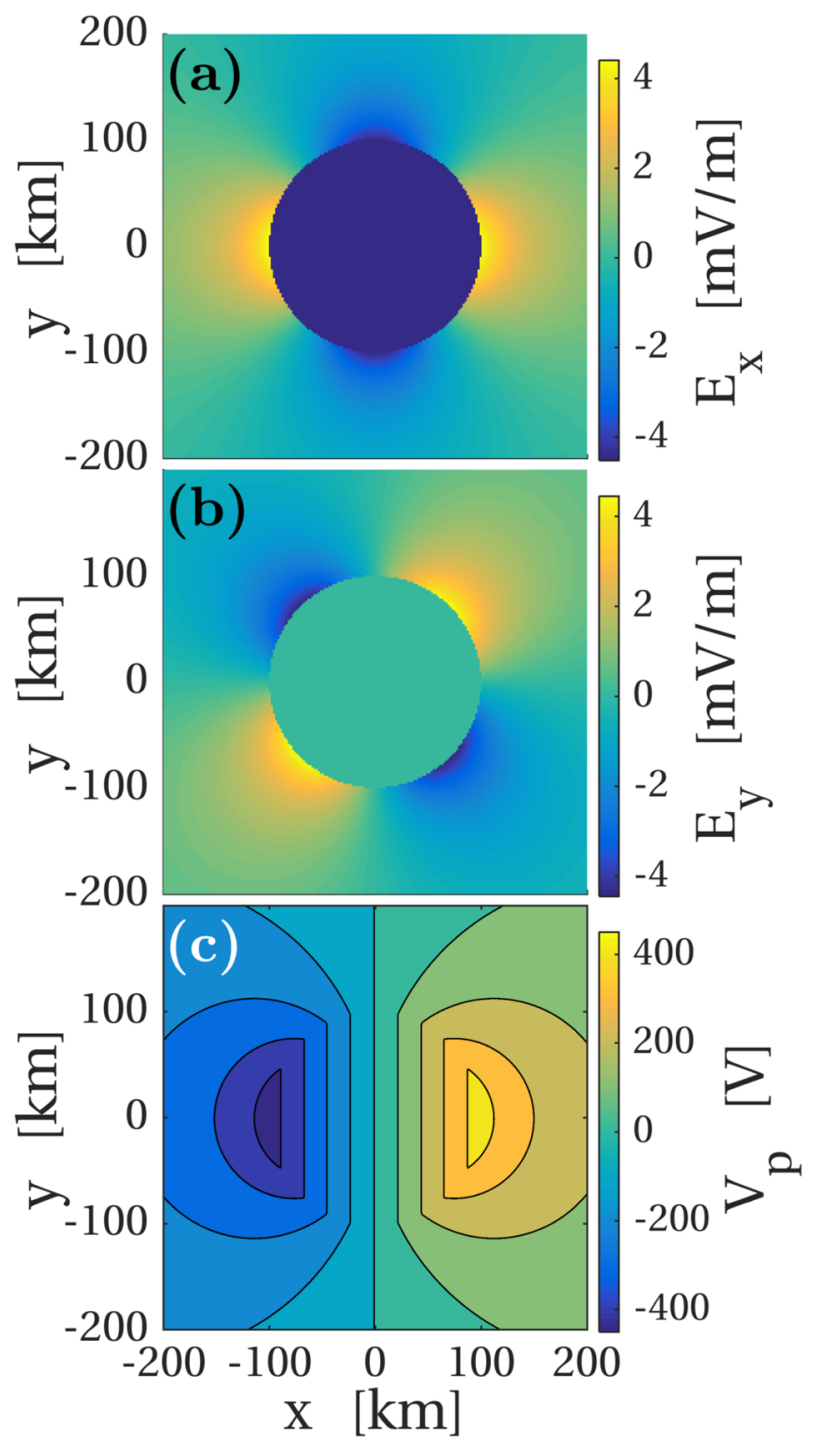

Fig. A.2. Electric field and potential in cylindrical geometry for a uniform plasma density as shown by the red curve in Fig. A.1. Panel a: $x$ component of the electric field. Panel $b: y$ component of the electric field. Panel $c$ : electrostatic potential $V_{\mathrm{p}}$.

boundary at $r_{\mathrm{c}}=d$ is shown by the black curve in Fig. A.1. The density is

$n_{\mathrm{e}}= \begin{cases}n_{0} \frac{R_{0}}{b} & \text { for } r_{\mathrm{c}} \leq b \\ n_{0} \frac{R_{0}}{r_{\mathrm{c}}} & \text { for } b<r_{\mathrm{c}} \leq d . \\ n_{0} \frac{R_{0}}{\mathrm{~d}} & \text { for } r_{\mathrm{c}}>d\end{cases}$

When displacing the ions with respect to the electrons in this case, the electric field can be obtained by a integrating the fields from an infinite number of concentric cylinders with a charge distribution along the $x$-axis as follows:

$\rho\left(x^{\prime}, 0\right)=e\left(n_{\mathrm{i}}-n_{\mathrm{e}}\right)= \begin{cases}0 & \text { for } x^{\prime} \leq b \\ \frac{e n_{0} R_{0} \Delta x}{x^{\prime}\left(x^{\prime}-\Delta x\right)} & \text { for } b<x^{\prime} \leq d . \\ 0 & \text { for } x^{\prime}>d\end{cases}$

The field at a given point $(x, y)$ can be written as a sum

$\boldsymbol{E}(x, y)=\boldsymbol{E}_{\mathbf{1}}(x, y)+\boldsymbol{E}_{\mathbf{2}}(x, y)$, 
where $\boldsymbol{E}_{\mathbf{1}}$ is the contribution from source outside a cylinder of radius $r_{\mathrm{c}}=\sqrt{x^{2}+y^{2}}$, and $\boldsymbol{E}_{\mathbf{2}}$ is the contribution from sources inside that same cylinder. The first contribution, $\boldsymbol{E}_{\mathbf{1}}$ comes from a superposition of cylinders larger than $r_{\mathrm{c}}$, as in Eq. (A.1), and only has an $x$ component

$$
\begin{aligned}
E_{x 1}\left(r_{\mathrm{c}}\right) & =\int_{r_{\mathrm{c}}}^{\mathrm{d}} \frac{e n_{0} R_{0} \Delta x}{2 \epsilon_{0} x^{\prime}\left(x^{\prime}-\Delta x\right)} \mathrm{d} x^{\prime} \\
& =\frac{e n_{0} R_{0}}{2 \epsilon_{0}}\left(\ln \left(1-\frac{\Delta x}{r_{\mathrm{c}}}\right)-\ln \left(1-\frac{\Delta x}{d}\right)\right) \\
& \approx-\frac{e n_{0} R_{0} \Delta x}{2 \epsilon_{0}}\left(\frac{1}{r_{\mathrm{c}}}-\frac{1}{d}\right) \quad \text { for } b<r_{\mathrm{c}} \leq d .
\end{aligned}
$$

For $r_{\mathrm{c}} \leq b$ we have

$$
E_{x 1} \approx-\frac{e n_{0} R_{0} \Delta x}{2 \epsilon_{0}}\left(\frac{1}{b}-\frac{1}{d}\right)
$$

For $r_{\mathrm{c}}>d$ there are no sources outside the field point, which ensures $E_{x 1}=0$ in that region. In the approximations above we used $\Delta x \ll r_{\mathrm{c}}$ and $\Delta x \ll b$.

The second term on the right-hand side of Eq. (A.5) stems from sources in the region $b \leq \sqrt{x^{\prime 2}+y^{\prime 2}} \leq r_{\mathrm{c}}$, and $\boldsymbol{E}_{2}$ is found by integrating contributions of the form given by Eq. (A.2) with respect to the cylinder radius. We define the vector $\boldsymbol{a}$, which depends only on the coordinates of the field point,

$\boldsymbol{a}=\left[\frac{x^{2}-y^{2}}{\left(x^{2}+y^{2}\right)^{2}}, \frac{2 x y}{\left(x^{2}+y^{2}\right)^{2}}\right]=\left[\frac{x^{2}-y^{2}}{r_{\mathrm{c}}^{4}}, \frac{2 x y}{r_{\mathrm{c}}^{4}}\right]$.

The electric field contribution $\boldsymbol{E}_{\mathbf{2}}$ for $b<r_{\mathrm{c}} \leq d$ then becomes

$$
\begin{aligned}
\boldsymbol{E}_{2}(x, y) & =\boldsymbol{a} \frac{e n_{0} R_{0} \Delta x}{2 \epsilon_{0}} \int_{b}^{r_{\mathrm{c}}} \frac{R^{\prime}}{R^{\prime}-\Delta x} \mathrm{~d} R^{\prime} \\
& =\boldsymbol{a} \frac{e n_{0} R_{0} \Delta x}{2 \epsilon_{0}}\left(r_{\mathrm{c}}-b+\Delta x \ln \left(\frac{r_{\mathrm{c}}-\Delta x}{b-\Delta x}\right)\right) \\
& \approx \frac{e n_{0} R_{0} \Delta x}{2 \epsilon_{0}}\left(r_{\mathrm{c}}-b\right) \boldsymbol{a} .
\end{aligned}
$$

The approximation relies on $\Delta x \ll r_{\mathrm{c}}$ and $\Delta x \ll b$. Inside $r_{\mathrm{c}}=b$ there is no net charge, and therefore

$$
\boldsymbol{E}_{\mathbf{2}}=0 \quad \text { for } r_{\mathrm{c}} \leq b,
$$

and outside the region where there are space charges we have

$$
\boldsymbol{E}_{2}(x, y) \approx \frac{e n_{0} R_{0} \Delta x}{2 \epsilon_{0}}(d-b) \boldsymbol{a} \quad \text { for } r_{\mathrm{c}}>d .
$$

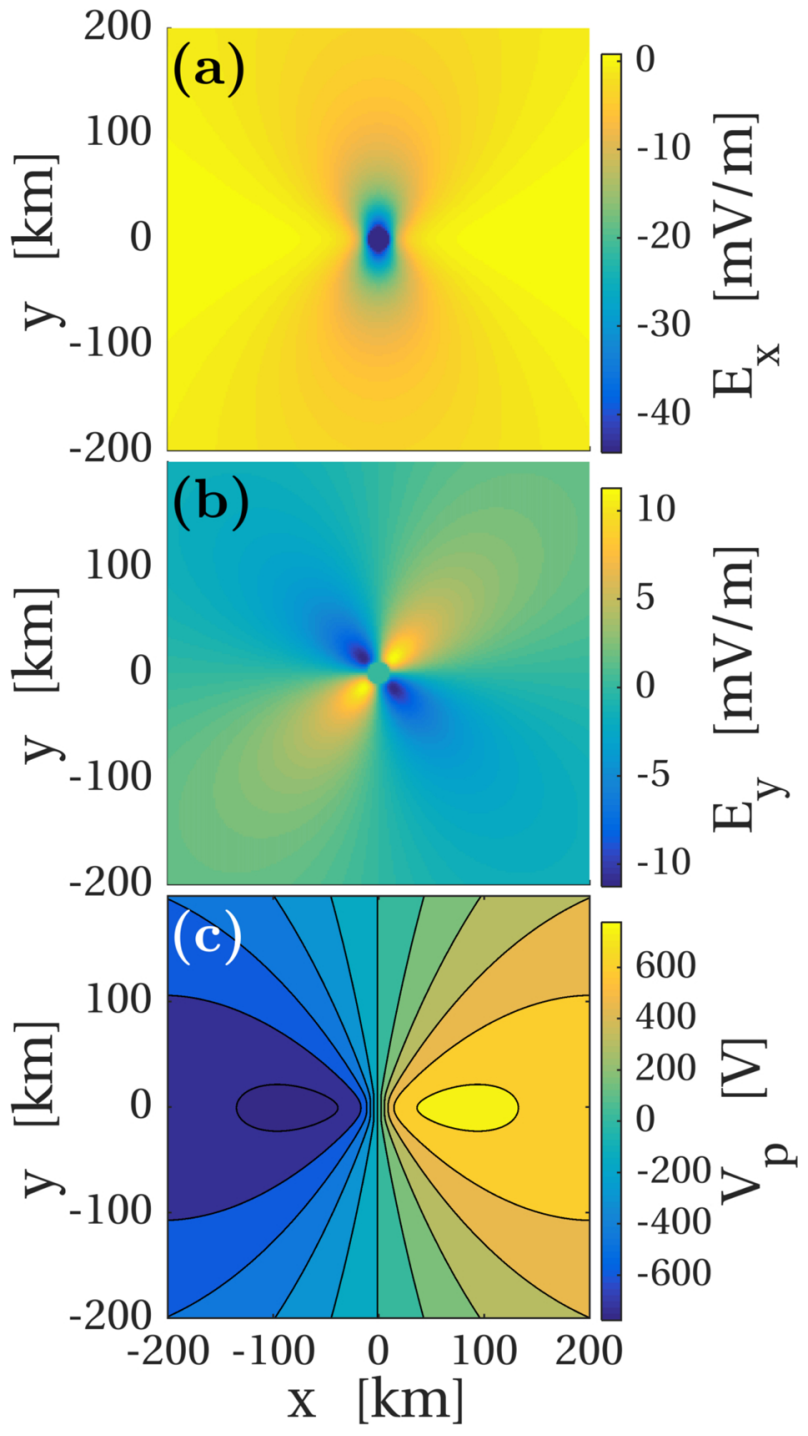

Fig. A.3. Electric field and potential in cylindrical geometry for a plasma density with a $1 / r_{\mathrm{c}}$ dependence as shown by the black curve in Fig. A.1. Panel $a$ : $x$ component of the electric field. Panel $b: y$ component of the electric field. Panel $c$ : electrostatic potential $V_{\mathrm{p}}$.

To summarise, the total field for small $\Delta x$ can be written

$$
\boldsymbol{E}= \begin{cases}-\frac{e n_{0} R_{0} \Delta x}{2 \epsilon_{0} b}\left(\frac{1}{b}-\frac{1}{d}\right)[1,0] & \text { for } r_{\mathrm{c}} \leq b \\ \frac{e n_{0} R_{0} \Delta x}{2 \epsilon_{0}}\left[\left(r_{\mathrm{c}}-b\right) \frac{x^{2}-y^{2}}{r_{\mathrm{c}}^{4}}-\frac{1}{r_{\mathrm{c}}}+\frac{1}{d}, \frac{\left(r_{\mathrm{c}}-b\right) 2 x y}{r_{\mathrm{c}}^{4}}\right] & \text { for } b<r_{\mathrm{c}} \leq d . \\ \frac{e n_{0} R_{0} \Delta x}{2 \epsilon_{0}}\left[(d-b) \frac{x^{2}-y^{2}}{r_{\mathrm{c}}^{4}},(d-b) \frac{2 x y}{r_{\mathrm{c}}^{4}}\right] & \text { for } r_{\mathrm{c}}>d\end{cases}
$$

This field is illustrated in Fig. A.3 for the parameters in Table A.1. 